\title{
功能模板导向的自组装合成介孔结构有机/无机 复合材料
}

\author{
李乐乐，孙聆东，张亚文，严纯华*
}

稀土材料化学及应用国家重点实验室, 北京大学-香港大学稀土材料和生物无机化学联合实验室, 北京大学化学与分子工程学院, 北京分 子科学国家实验室, 北京 100871

* 通讯作者, E-mail: yan@pku.edu.cn

收稿日期: 2009-09-21; 接受日期: 2009-09-27

摘要回顾了近年来硅基介孔材料有机功能化的基本方法和研究进展. 基于作者 的相关研究工作, 着重介绍一种新型的介孔氧化硅有机功能化的方法——功能模板 导向的自组装法，阐述了该方法在自组装合成新型有机/无机复合材料方面的应用.

\section{关键词} 介孔材料

自组装 有机-无机复合材料

\section{1 引言}

Science 杂志于 2005 年载文指出, 如何推动化学 自组装的发展是化学家在 21 世纪面临的 25 个核心科 学问题之一1]. 所谓自组装是指结构单元通过非共价 键相互作用 (如氢键、 $\pi-\pi$ 堆积、静电、疏水作用等) 自发地形成热力学稳定、结构确定、具有某种特定功 能聚集体的过程 ${ }^{[2-4]}$. 自组装合成具有特定结构和功 能的新材料已成为化学和材料科学等领域的重要方 法，具有其它手段难以取代的优越性.

自从表面活性剂为模板的自组装方法在 1992 年 被美国 Mobil 公司的科学家成功用于有序介孔氧化 硅的合成以来 ${ }^{[5]}$, 以表面活性剂等为模板, 通过自组 装合成介孔材料研究就受到了人们的高度重视, 并迅 速成为跨学科的研究热点之一. 用于介孔材料合成的 双亲性表面活性剂种类很多, 如阳离子型长链烷基季 铵盐、聚氧乙烯-聚氧丙烯共聚物(PEO-PPO)和三嵌段 共聚物(PEO-PPE-PEO)等. 所合成的有序介孔材料具 有孔道尺寸均匀、排列有序、孔径可调等特性, 在催 化、能源、环境、生物和光电转换等领域显示了极为 重要的应用价值 ${ }^{[6-8]}$. 经过近二十年的研究, 多种孔径
可调、组成可变、形貌和孔道结构多样化的新型硅基 介孔材料已被成功合成. 与此同时，介孔单质、介孔 金属氧化物、介孔硫化物和介孔磷酸盐等非硅基介孔 材料也相继问世 $\stackrel{[9 \sim 16]}{ }$.

自人工合成介孔材料问世以来, 关于其形成机理 问题一直是人们所讨论的热点, 其中液晶模板机理 (liquid-crystal templating mechanism, LCT) ${ }^{[5,17]}$ 和协同 组装机理(cooperative formation mechanism, CFM) $)^{[18]}$ 是两种最具代表性的组装机理. 如图 1 所示, 在液晶 模板机理中, Mobil 公司的科学家认为表面活性剂在 无机物加入前, 首先在溶液中形成疏水端在胶束内 核, 而亲水端暴露在外的球形胶束; 然后形成棒状胶 束; 当表面活性剂浓度足够高时自发形成六方有序 排列的液晶结构. 加入的无机硅源与表面活性剂的亲 水端具有较强的作用力, 因此进而可以沉积在棒状胶 束的周围, 并进一步聚合固化成孔壁(图 1(a)途径) $\frac{[5,17]}{\text {. }}$ LCT 机理的提出借鉴了表面活性剂的液晶形成过程, 第一次阐明了介孔分子篎合成中的“模板”概念，为人 们理解介孔分子篮的形成提供理性指导. 另一方面, Stucky 小组提出的协同组装机理(CFM) 具有一定的普 


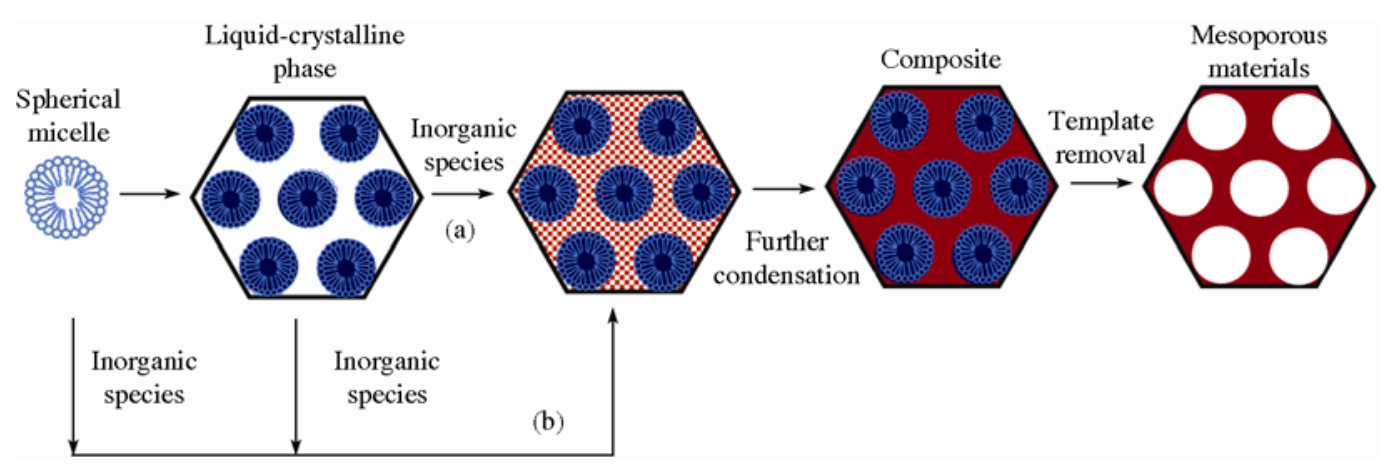

图 1 介孔材料的形成机理

(a)液晶模板机理和(b)协同组装机理

适性, 是目前广为接受的介孔材料形成机理 ${ }^{[18]}$. 与 LCT 机理不同的是, CFM 机理认为表面活性剂的液 晶相是在加入无机反应物后形成的, 无机物种与表面 活性剂分子间通过协同作用和界面电荷匹配自组装 形成有序介观结构 (图 1(b)).

介孔氧化硅作为一种新型的无机多孔材料, 具有 有序可调的纳米孔道、高的热稳定性、大的比表面等 诸多优点, 受到了大家的广泛关注. 然而, 对于纯硅 基介孔材料而言, 其骨架全部由氧化硅构成, 本身并 没有活性中心，这就限制了它的实际应用. 对于它在 应用方面的开发是目前介孔氧化硅材料研究中的热 点之一. 事实表明, 介孔物质是组装化学中的优良主 体材料, 为客体分子的固载与结构控制提供了基础. 为了开发具有特定性质的硅基介孔功能材料, 科学家 们发展了多种方法用以实现硅基介孔材料的有机功 能化, 从而获得具有特殊功能的有机/无机介孔复合 材料. 有机功能基团的引入可以赋予介孔二氧化硅具 有离子吸附、催化、光电转换和响应等多种功能, 同 时, 无机孔道结构还可对限域的有机客体分子起到稳 定和保护作用. 基于介孔二氧化硅的结构和孔道表面 特性, 以及有机模板导向自下而上的自组装机理, 目 前主要有后嫁接法(post-synthetic grafting technique)、 共缩聚法(co-condensation method)和周期性介孔有机 硅的合成(preparation of periodic mesoporous organosilicas)等 3 种方法用以实现它的有机功能化 ${ }^{[19-26]}$. 本 文将简单介绍这 3 种有机功能化方法及其相应的特 点, 同时, 基于本组近年来的一些研究工作, 还总结 提出了一种新型的介孔二氧化硅有机功能化的方法,
即功能模板导向的自组装法 (functional-template directed self-assembly, FTDSA). 作者认为该方法在合 成介观尺度有序的有机/无机复合材料方面将具有良 好的应用潜力。

\section{2 介孔氧化硅的三种基本有机功能化方法}

后嫁接法主要利用了介孔二氧化硅比表面积大、 表面富含的硅羟基可以提供有机杂化反应活性位点 的特色. 后嫁接法属于介孔氧化硅制备后的化学修饰 方法, 即先制备介孔氧化硅, 然后在其表面共价连接 有机分子. 如图 2 所示, 该方法过程简单, 一般是将 活泼的有机硅烷偶联剂 $\left(\mathrm{R}^{\prime} \mathrm{O}\right)_{3} \mathrm{Si}-\mathrm{R}$ 在惰性有机溶剂 中与介孔氧化硅材料反应, 由于有机硅烷偶联剂的烷 氧基团( $\left.\mathrm{R}^{\prime} \mathrm{O}\right)$ 水解后生成的硅醇键 $\mathrm{Si}-\mathrm{OH}$ 可与无机物 表面的羟基通过缩聚反应形成牢固的化学键, 因而可

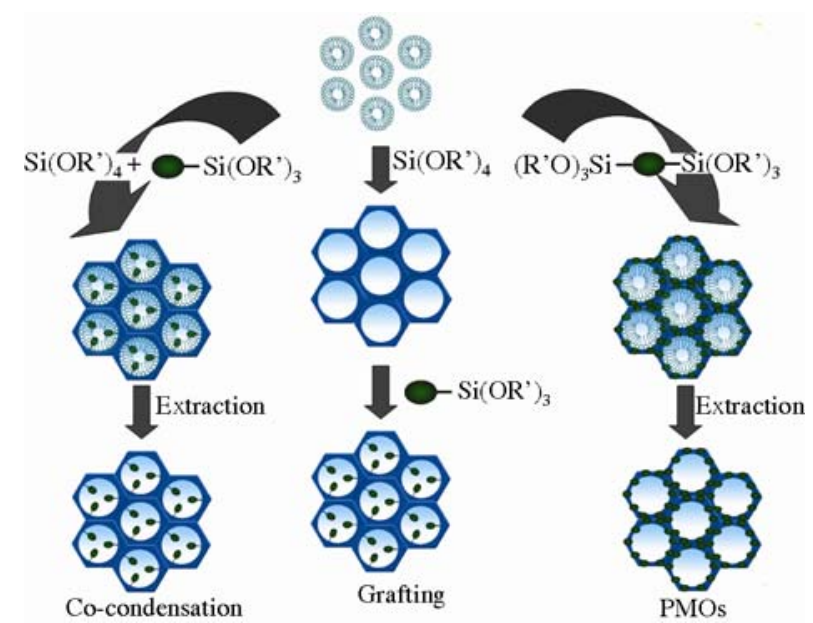

图 2 介孔二氧化硅材料有机功能化方法示意图 22$]$ 
将不同有机官能团 $\mathrm{R}$ 共价连接在介孔二氧化硅的表 面. 原理上，通过合成含有不同有机官能团的烷氧基 硅烷或卤代硅烷等有机硅烷偶联剂, 就可以将各种有 机功能团嫁接到孔道表面. 有机硅化学的发展为该方 法提供了可行的化学途径. 在一步嫁接反应后, 利用 被接枝功能团的反应性功能, 可以继续对嫁接的有机 功能团改性或者接枝. 如嫁接到孔道内的氧基可被水 解为羧酸, 嫁接的颈基可以进一步被氧化为磺酸基等. 经过嫁接反应后, 功能化介孔材料的结构有序性一般 可以得到很好的保持. 该方法中, 有机功能团的嫁接 需要其首先扩散进孔道内部, 且与二氧化硅表面的羟 基发生缩聚反应. 因此, 该方法具有一定的不足：(1) 有机功能团的嫁接含量较低; (2) 有机功能团在孔道 内的分布不均匀, 其更易于在孔道口处嫁接, 这会增 加有机硅烷偶联剂进入孔道深处进行反应的难度, 导致在孔道深处嫁接含量相对要少, 甚至无法嫁 接 $[21,22]$.

如图 2 所示, 共缩聚法属于“一锅法”, 是直接在 合成介孔二氧化硅的过程中加入一定比例的有机硅 烷偶联剂, 使之作为部分的硅源在有机模板导向下进 行自组装和缩聚反应. 自组装过程中, 含有疏水功能 团的有机硅烷偶联剂可起到共模板作用, 使有机功能 团插入表面活性剂形成的胶束中心, 所以能够得到官 能团分布较均匀的功能化介孔二氧化硅 ${ }^{[22]}$. 为了保持 复合的有机功能团的完整性, 避免 $\mathrm{Si}-\mathrm{C}$ 键的断裂, 一 般要采用萃取法而不是焙烧来除去模板剂. 与后嫁接 法相比, 该方法操作步骤简单, 同样可以将多种有机 官能团固定在介孔材料表面, 而且有机功能团的分布 比较均匀. 但是有机功能团的复合含量一般不能超过 $25 \%$, 如果前驱体中加入过多的硅烷偶联剂, 会影响 自组装过程, 导致介孔材料有序结构的破坏 ${ }^{[21]}$. 后嫁 接和共缩聚两种方法已被广泛用于将特定有机基团 修饰到介孔氧化硅的表面, 从而获得具有特定性质的 功能材料. 这些有机基团可以是简单的烷基、巯基和 胺基等, 也可以是具有光、电、磁性质的特殊功能团. 所得复合材料在催化、吸附和分离、药物的可控释放、 光学、电学等方面具有重要的应用潜力, 有望在催化 工业、环境工程、生物医药和光电子器件等方面得到 商业化发展

与上述两种介孔氧化硅材料的有机功能化方法
不同的是，周期性介孔有机硅(PMOs)是指有机基团 存在于介孔材料孔壁中的有机/无机复合材料. 它是 由 Inagaki 小组 ${ }^{[35]}$ 、Ozin 小组 ${ }^{[36]}$ 和 Stein 小组 ${ }^{[37]}$ 于 1999 年相继独立报道的. 如图 2 所示, PMOs 是由桥键型 有机硅烷偶联剂 $\left(\mathrm{R}^{\prime} \mathrm{O}\right)_{3} \mathrm{Si}-\mathrm{R}-\mathrm{Si}\left(\mathrm{OR}^{\prime}\right)_{3}$ 作为硅源, 在有 机模板的导向作用下自组装合成的. 由于使用的桥键 型硅源两端都可以水解交联, 参与无机骨架网络的构 建，从而实现有机功能团 $\mathrm{R}$ 均匀分布于骨架中. 在自 组装过程中, 桥键型有机硅烷偶联剂可以作为唯一的 硅源, 因而有机功能团的复合含量可达到 $100 \%$. 如使 用 $\mathrm{R}$ 为共轭芳香环的桥键型有机硅烷偶联剂作为硅 源合成 PMO, 有机官能团苯环通过较强的 $\pi-\pi$ 相互作 用排列成有序的层状结构来构成孔壁, 可以得到具有 类晶体结构孔壁的分子篮 ${ }^{[38,39]}$. 值得一提的是, 由于 PMOs 中的有机基团均匀分布于无机骨架内, 因此, 即使含量很高也不会阻塞孔道和占据孔容, 这将有利 于客体分子扩散到活性位点进行反应. PMOs 的结构 与性能取决于有机硅前驱体中有机桥联基团的种类, 功能团的引入还可以提高材料的机械强度和热稳定 性, 因而可以通过焙烧或萃取方法脱除表面活性剂模 板. 均匀嵌入介孔壁内的有机官能基团由于所处的环 境不同, 还将表现出不同的化学特性, 从而赋予材料 可控的化学和物理性能 [24 26]. 此外, 孔壁中的有机基 团还可以继续反应, 衍生出新的活性中心. 但是, 有 机功能基团常常由于氢键、静电等弱作用和空间位阻 等原因影响表面活性剂的有序组装以及水解缩合过 程, 易形成不具备有序孔道结构的材料. 迄今为止, 许 多 PMOs 已被合成, 但嵌入到介孔壁内的有机官能团 仍局限于一些简单的有机基团, 如亚甲基、乙烷、乙 烯基和苯等. 为了拓展其功能、提高实际应用价值, 人 们正致力于将具有吸附性 ${ }^{[40,41]}$ 、光学 ${ }^{[42-44]}$ 、电学 ${ }^{[45,46] 、}$ 手性 $\stackrel{[47,48]}{ }$ 和催化 4 [49,50]等性质的特殊有机功能团引入 PMOs, 旨在获得特定功能有机/无机复合材料.

\section{3 功能模板导向的自组装法}

\section{1 功能模板导向的自组装合成有序高分子/氧化 硅复合材料}

表面活性剂在工业生产和日常生活中有着广泛 应用. 表面活性剂在分子结构上的共同特点是它们 都是由亲水的极性基和亲油的非极性基构成的，因 而在不同极性的溶剂中能自发形成有序的超分子聚 
集体. 表面活性剂为模板的自组装方法被用于合成 有序介孔材料以来，该自组装思想受到科学家们的 广泛关注. 在这种方法中, 表面活性剂并不具备特殊 的功能性, 只起到了导向生成介孔结构的模板作用. 而在我们定义的功能模板导向的自组装法中, 双亲 性的表面活性剂不仅仅起到生成有序无机材料的结 构导向剂作用, 它本身还具有特殊的功能性, 因而通 过一步的自组装过程, 就可以得到高度有序的有机/ 无机杂化材料.

功能模板导向的自组装思想最早被用于一步法 制备有序的高分子/氧化硅复合介孔材料. 该方法的 关键是设计合成特殊的双亲性表面活性剂, 即所用表 面活性剂不仅可以作为形成介观结构的结构导向剂 (structure-directing agents), 其本身还是聚合生成高分 子的单体(monomer). Brinker 等 ${ }^{[51,52]}$ 首先报道了利用 功能模板导向的自组装方法制备高度有序高分子/氧 化硅介孔复合材料 (图 3). 他们采用含有联乙炔单体 的两亲性表面活性剂作为结构导向剂, 通过溶剂挥发 诱导自组装方法制备了介观有序的复合薄膜, 实现了 联乙炔单体在氧化硅纳米孔道内的可控排列. 他们通 过调节两亲性表面活性剂分子亲水链段的长度, 制备 了层状、六方和立方介观相的复合材料. 分子模拟表 明, 联乙炔单体在氧化硅纳米孔道内具有分子水平上 有序的排列和取向. 利用紫外光引发不饱和联乙炔单 体的聚合, 进而得到了以聚二乙炔为骨架的共轭聚合 物. 该高分子/氧化硅复合材料表现出优异的光学性 能和环境响应功能. Aida 小组 ${ }^{[53]}$ 以含有联乙炔单体的 阳离子表面活性剂为结构导向剂, 制备了具有光致发 光特性的聚二乙炔/氧化硅复合材料. 该材料具有微 米级的棒状形貌, 因而可以方便地在光学显微镜下对 它进行操作, 从而测试其光学和电学性质.

功能模板导向的自组装方法在合成有序高分子/ 氧化硅复合材料中已得到广泛应用. Aida 等以疏水链 段连有吡咯基团的阳离子表面活性剂为模板, 制备了 介观有序的复合材料薄膜, 进而以 $\mathrm{FeCl}_{3}$ 为氧化剂使 氧化硅纳米孔道内的吡咯基团氧化聚合, 得到聚吡咯 /氧化硅复合薄膜 ${ }^{[54]}$. 通过同样的方法, $\mathrm{Li}$ 等合成了连 有吡咯基团的离子液体型表面活性剂, 通过一步自组 装方法实现了聚吡咯钠米线在 MCM-41 氧化硅纳米 孔道内的排列 ${ }^{[55]}$. 上述报道中所得到的材料都是有机/
无机复合材料, 是否可将复合材料中的氧化硅除去, 从而得到高分子纳米材料呢? Fuhrhop 小组的研究表 明, 功能模板导向的自组装方法还可以用于合成高分 子纳米材料 (图 4) ${ }^{[56]}$. 他们以含有噻吩的阳离子表面 活性剂为结构导向剂, 通过一步法在碱性条件下制备 了聚噻吩/氧化硅复合材料, 去除氧化硅后成功地得 到了聚噻吩的纳米线. 当在溶液中加入 $\mathrm{PF}_{6}{ }^{-}$离子与高 分子相互作用后, 还可使聚噻吩纳米线整齐排列起 来.

最近, Wang 等利用功能模板导向的自组装方法 在酸性条件下制备了不同形貌的聚噻吩/氧化硅复合 材料 ${ }^{[57]}$. 他们合成了两种不同疏水链长的连有噻吩单 体的阳离子表面活性剂, 分别将其作为结构导向剂, 制备了分别具有六方有序孔道和蠕虫状孔道结构的 复合材料. 该材料可发射出强而稳定的荧光. 由于聚 噻吩链在六方有序孔道中要比在蠕虫状孔道中具有 更高的有效共轭长度, 其吸收和苂光光谱发生了明显 红移. Tolbert 等使用一种两亲性半导体高分子(聚苯 撑乙烯)和阳离子表面活性剂 CTAB 作为共结构导向 剂, 得到的高分子/氧化硅复合材料保持了聚苯撑乙 烯的光物理性质, 且表现出单链的光学行为 ${ }^{[58]}$.

\section{2 功能模板导向的自组装法合成光电复合材料}

功能模板导向的自组装法中, 作为结构导向剂 的功能性表面活性剂不仅可以连接可聚合成高分子 的单体、获得有序结构高分子/氧化硅复合材料, 还可 以携带具有特定光电性质的有机功能团, 通过一步 自组装过程获得纳米尺度有序的光电功能材料. 作 者将功能模板导向的自组装方法进一步发展, 利用 其实现光电小分子在无机纳米孔道内的有序组装, 获得了对外界刺激 $(\mathrm{pH})$ 响应的纳米复合材料 ${ }^{[59]}$. 如 图 5 所示, 我们设计合成了两个在疏水链端连有不同 光电小分子的阳离子表面活性剂, 进而利用它们作 为结构导向剂和功能构建单元的双重特性, 制备了 介观有序的纳米复合材料. 利用在疏水链端连接具 有光电活性的 5-甲氧基-2-苯基噻唑小分子的阳离子 表面活性剂为结构导向剂, 所得微米颗粒状复合材 料具有二维六方有序孔道结构. 而利用连接 5-甲氧 基-2-吡啶噻唑小分子的阳离子表面活性剂合成的复 合材料具有亚微米尺寸的球状外形和蠕虫状孔道结 


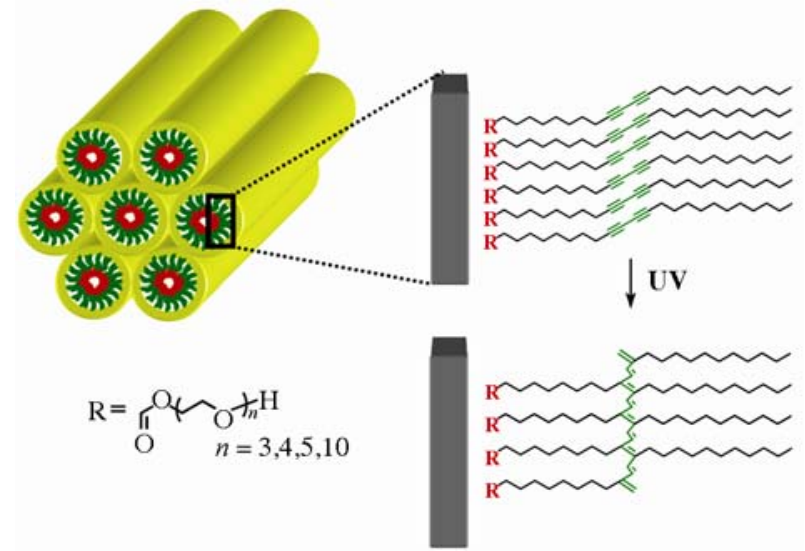

图 3 功能模板导向的自组装合成聚二乙炔/氧化硅纳米 复合材料 $[52]$
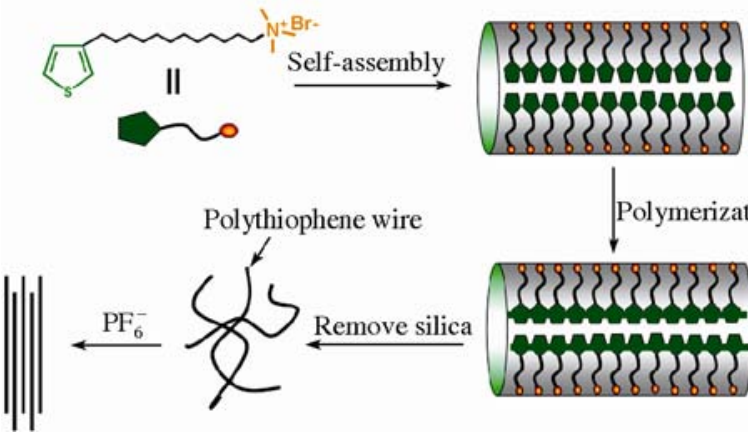
Polymerization

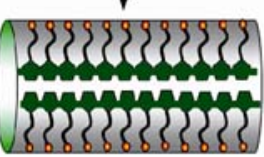

图 4 功能模板导向的自组装合成聚噻吩高分子纳米线 [56]

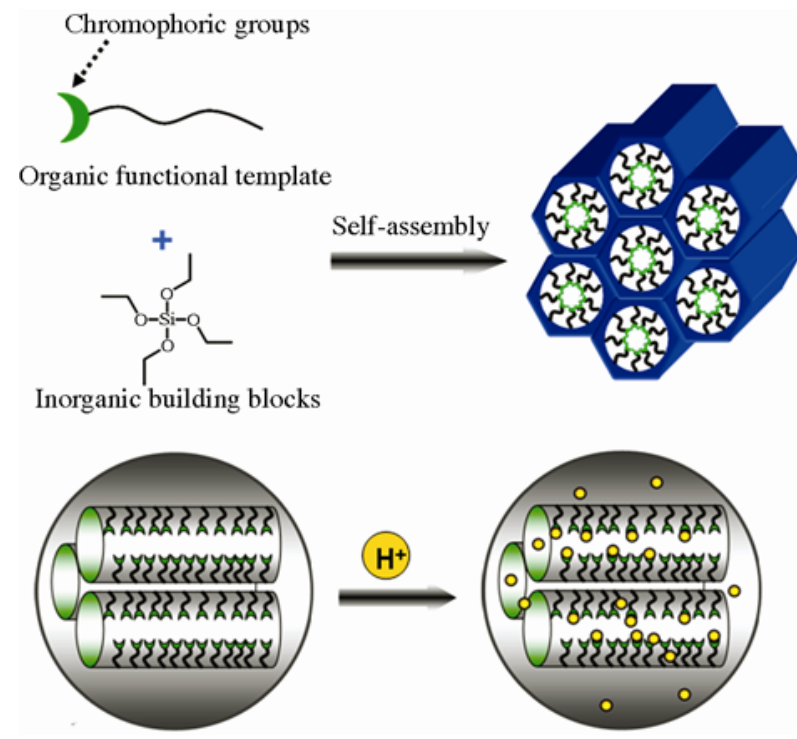

图 5 功能模板导向的自组装合成对外界刺激 $(\mathrm{pH})$ 响应的 纳米复合材料 [59]
构. 这两种光电复合材料都具有强的荧光发射，基于 所用光电功能有机小分子的不同特性，它们分别具 有随 $\mathrm{pH}$ 改变而线性变化的荧光发射强度和可用于双 发射波长比率测量的 $\mathrm{pH}$ 苂光传感行为. 通过自组装 过程获得光电小分子在无机纳米孔道内的有序组装, 不仅可以提高复合材料的机械强度和热稳定性，还 可以提高 $\mathrm{pH}$ 光学传感的灵敏性. 这类可用于水相 $\mathrm{pH}$ 检测的荧光传感器有望应用于分析化学、生命科学和 环境监测等领域，这一自组装思想还可以拓展应用 于其它光电传感器的制备.

有机半导体材料和器件研究是当今热点课题之 一. 为了提高其应用性能, 需要充分增强半导体分子 间的电子传递能力. 实现功能分子的规则有序排列 是提高其电子传输能力的关键之一. 然而, 如何有效 地通过分子设计和合成手段，调控有机半导体材料 的空间排列行为, 理解可控组装及其构效关系仍是 一项挑战性的工作. 最近，作者利用功能模板导向的 自组装方法, 实现了有机半导体分子(蒽)在氧化硅周 期性纳米孔道内的定向排列和对分子光物理性质的 调控 ${ }^{600]}$. 如图 6 所示, 我们利用在疏水链端键连葱分 子的阳离子表面活性剂为结构导向剂, 通过一步自 组装法合成了有序介孔复合材料. 有机半导体纳米 材料制备中，通常以共轭半导体分子间的 $\pi-\pi$ 作用实 现其组装、实现有机半导体分子的规则排列. 而在我 们的工作中, 则是是利用两亲性有机半导体分子与 无机物种间的相互作用，通过自组装过程取向排列 葸分子. 该方法不仅可以实现有机功能分子在无机 孔道中的高含量复合和均匀分布, 还能在分子水平 上操控它们的空间聚集态行为，这就为研究其分子 堆积结构与性质之间的关系提供了条件. 此外, 均匀 定向排列在纳米孔道中的葱分子还显示出特殊的光 电性质，与无序的葱分子发射出单体(monomer)的苂 光行为相比, 葱分子在氧化硅纳米孔道内定向排列 后，由于分子间产生强烈的 $\pi-\pi$ 相互作用，因而发射 出独特的激基缔合物（excimer）的荧光. 这表明该组 装可以有效的调控和裁剪有机半导体的光物理和光 化学性质，为拓展相关材料的应用提供了可能. 作者 认为，该方法可以被推广应用于其它有机半导体分 子的可控组装及其复合材料制备，也为开发相关光 电器件提供了平台. 


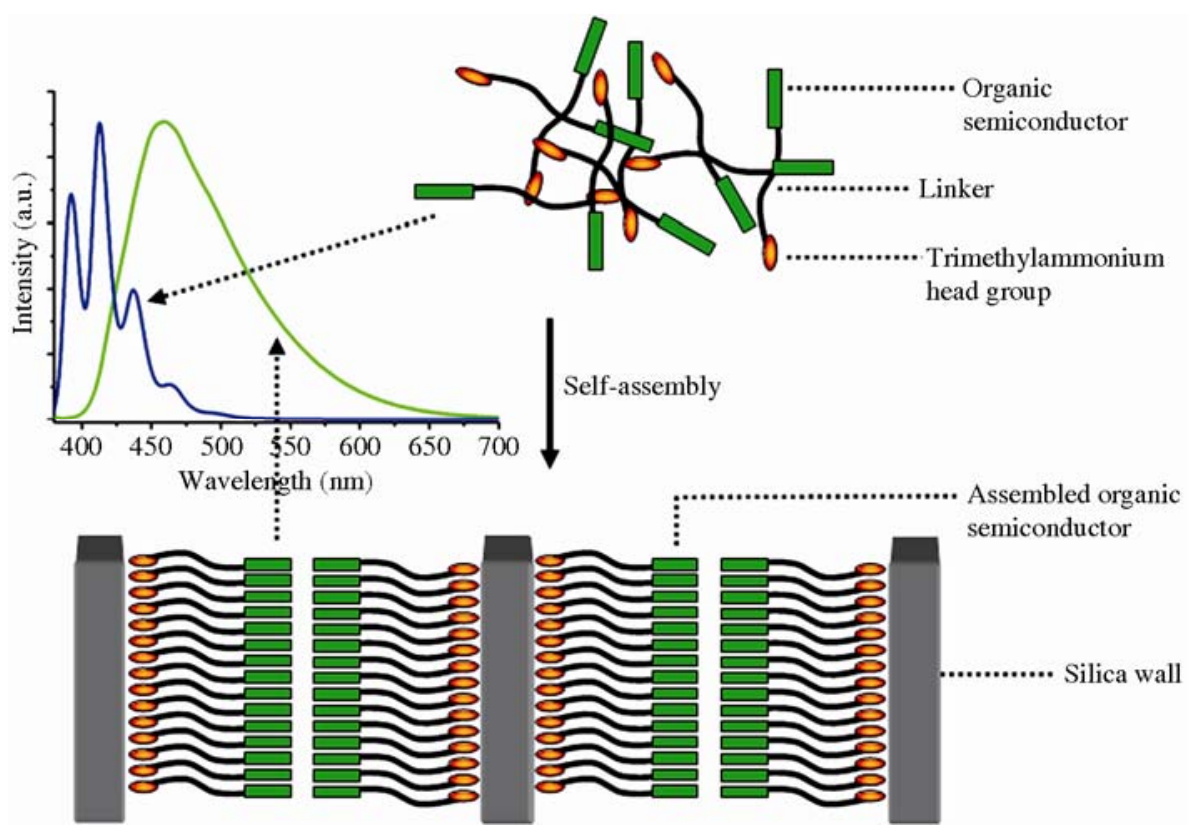

图 6 功能模板导向的自组装法调控有机半导体的空间排列及光物理性质 60$]$

光电功能材料的多功能化可以丰富和扩展材料 的应用范围. 作者成功将功能模板导向的自组装方 法推广应用于介孔结构双功能材料的可控合成和组 装中 ${ }^{[61]}$. 如图 7 所示, 为了实现材料的多功能化, 必 须设计和合成两个带有不同功能团 $\mathrm{A}$ 和 $\mathrm{B}$ 的自组装 构建单元. 将疏水链端连有一个功能基团 $\mathrm{A}$ 的阳离
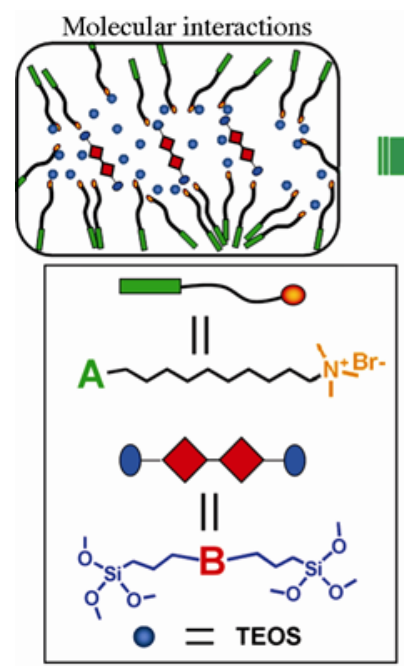
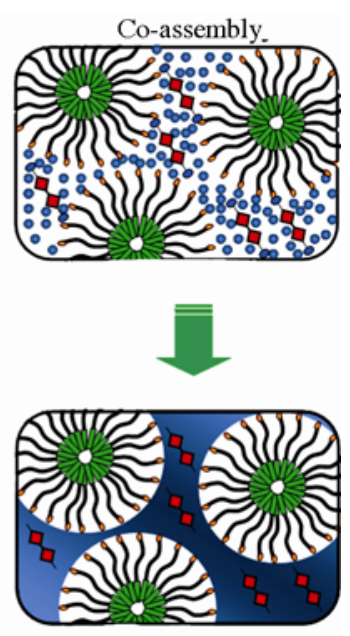

Condensed hybrid
图 7 拓展的功能模板导向的自组装法通过将两个不同功 能团 $\mathbf{A}$ 和 $\mathbf{B}$ 均匀排列在介孔材料的孔道和孔壁中来制备多 功能材料 $[61]$
子表面活性剂作为合成介孔材料的结构导向剂，另 将桥键型有机硅烷偶联剂 $\left(\mathrm{R}^{\prime} \mathrm{O}\right)_{3} \mathrm{Si}-\mathrm{B}-\mathrm{Si}\left(\mathrm{OR}^{\prime}\right)_{3}$ 作为 生成无机骨架的硅源，通过一步自组装过程就可以 分别将功能团 $\mathrm{A}$ 和 $\mathrm{B}$ 均匀排列在复合材料的孔道和 孔壁中，从而实现功能复合. 显然，通过设计不同性 质的功能团 $\mathrm{A}$ 和 $\mathrm{B}$, 结合相应自组装构建单元的合成, 就可以构筑多种双功能材料. 该方法可以被理解为 功能模板导向的自组装法合成周期性介孔有机硅 (PMOs). 作为该方法的实践，作者合成了三维有序 的电子给体-受体纳米复合材料 ${ }^{[61]}$. 该方法可以拓展 到其它光电复合材料的合成，为场效应晶体管、太阳 能电池等器件的制备提供新的研究思路.

$\mathrm{Hsu}$ 等通过溶剂挥发诱导的自组装技术制备了 氧化硅基光电复合薄膜材料. 他们以含有染料发色 团的两亲性分子在自组装过程中为有机模板，实现 了染料分子在氧化硅纳米孔道中的堆积 ${ }^{[62,63]}$. 研究 表明，这些表面活性剂染料结合于孔道中后，荧光发 射增强、荧光量子效率显著提高. 他们认为这是由于 染料在孔道中定向有序排列，从而减少荧光自猝灭 的结果. 迄今, 功能模板导向的自组装方法主要应用 于氧化硅基复合材料的合成. 最近, Stupp 组将功能 模板导向的自组装方法拓展到了非硅基 $(\mathrm{ZnO})$ 有序介 
孔复合材料的制备 ${ }^{[64]}$. 作为结构导向剂的功能两亲 性分子不仅为复合材料引入了光电功能团, 还稳定 了层状结构的 $\mathrm{Zn}(\mathrm{OH})_{2}$ 向半导体 $\mathrm{ZnO}$ 的转变. 该复合 材料具有稳定的光导性质，同时，有序层状结构中的 有机和无机层都可以有效地传递电子, 因而为开发 新型光伏器件提供了新思路.

\section{4 总结和展望}

人类社会的进化与材料的发展总是同步进行的， 新材料的诞生必然影响了社会的进步和变革. 自组装 技术已成为功能材料合成的最重要的方法之一. 在自 组装方法合成介孔材料方面，除了继续研究和开发材 料的绿色、可控制备新方法外，如何以功能为导向、 以需求为目标, 开发新型多功能复合材料, 将基础研究 结果转化为实际可用技术, 这是该领域的发展关键.

硅基介孔材料的有机功能化为有机/无机复合材 料的制备及应用开辟了新的途径. 目前，硅基介孔材 料的有机功能化主要通过后嫁接法、共缩聚法和周期 性介孔有机硅的合成得以实现. 简单而言，前两种方 法是对孔道的功能化，而第三种方法是对孔壁的改性. 功能模板导向的自组装法是一种介孔氧化硅有机功 能化的新方法，也是对上述 3 种合成方法的发展. 目 前, 对这一方法的研究和应用仍处于起步阶段, 发展 空间较大. 本文通过它在有序高分子/氧化硅复合材 料及光电功能材料合成中应用实例，展示了该方法在 自组装合成新型复合材料方面的特点和重要作用. 利 用该方法所取得的初步结果是可喜的，然而该方法也 具有一定的缺点和挑战. 其中, 具有结构导向作用和 功能构建单元双重特性的有机功能模板的设计和合 成是该方法的关键，但它的合成一般需要多步有机反 应，导致费时且增加成本. 同时，最终的自组装过程 对很多实验参数都比较敏感, 因此对其调控也相对比 较复杂. 随着对介孔材料的深入研究和有机合成技术 的进步，功能模板导向的自组装法合成有机/无机纳 米复合材料仍然有很大的发展潜力. 基于这一方法的 理解，作者认为它将在以下 5 个方面的研究中发挥重 要作用.

(1) 合成具有特定功能的高分子/氧化硅纳米材
料. 可以通过设计可聚合高分子单体，首先合成两亲 性表面活性剂结构, 进而以它为结构导向剂, 与无机 结构单元进行自组装，制备有序介孔复合材料，实现 单体在氧化硅纳米孔道内的可控排列和堆积. 进一步 利用外界条件引发单体聚合, 获得具有特定光学、电 学或磁学等性质的高分子/氧化硅复合材料. 通过去 除复合材料中的氧化硅, 还可以应用于高分子纳米线 的合成, 这就为高分子纳米功能材料的制备提供了新 的途径.

（2）构建光学传感器和药物输运体系. 利用功能 模板导向的自组装方法, 将对外界刺激具有响应能力 的光电分子组装, 可望得到光学传感材料. 若组装于 孔道内的功能分子可在适当的外界刺激下控制释放， 就有可能将小的药物分子利用功能模板导向的自组 装方法复合于材料的孔道内, 进而实现药物输运和可 控释放.

（3）制备具有导电性质的材料和器件. 功能模板 导向的自组装方法可以有效地实现有机半导体小分 子或导电高分子在纳米孔道内的限域和紧密排列。 这种纳米尺度上的组装为研究功能分子的光电性质、 制备相关的微纳器件提供了新的机会. 由于纳米尺 度上有序的孔道结构并不具有宏观取向性，因此，对 导电性质的测试仍是一个挑战. 薄膜技术的发展将 为实现该类材料孔道的定向排列、解决导电性质的测 试难题提供了可能.

（4）制备多功能纳米材料. 将功能模板导向的自 组装法与后嫁接法、共缩聚法、以及周期性介孔有机 硅的合成方法相结合，可用于介观结构多功能材料 的可控合成和组装. 若将功能模板导向的自组装法 合成周期性介孔有机硅(PMOs), 就有可能在孔道和 孔壁中分别引入不同的功能团，实现多种功能在同 一种材料中的集成.

(5) 非硅基纳米复合材料的拓展. 染料敏化太阳 能电池结合了光敏染料和无机半导体的优势, 两者的 组装和界面作用对其能带配合、载流子分离和输运, 以及电池性质具有关键作用. 利用连有光敏染料的表 面活性剂作为结构导向剂, 合成有序光敏染料/介孔 $\mathrm{TiO}_{2}$ 复合材料, 有望得到优良的光伏电池等器件.

致谢本工作得到了国家自然科学基金(批准号: 20821091, 20771009)资助，特此致谢。 


\section{参考文献}

1 Service R F. How far can we push chemical self-assembly? Science, 2005, 309: 95

2 Whitesides G M, Grzybowski B A. Self-assembly at all scales. Science, 2002, 295: 2418-2421

3 Lehn J M. Supramolecular chemistry: Concepts and perspectives. Germany: VCH, Weinheim, 1995

4 Ringsdorf H, Simon J. Molecular self-assembly-snap-together vesicles. Nature, 1994, 371: 284-284

5 Beck J S, Vartuli J C, Roth W J, Leonowicz M E, Kresge C T, Schmitt K D, Chu C T W, Olson D H, Sheppard E W, McCullen S B, Higgins J B, Schlenker J L. A new family of mesoporous molecular sieves prepared with liquid crystal templates. J Am Chem Soc, 1992, 114: 10834-10843

6 Zhao D Y, Feng J L, Huo Q S, Melosh N, Fredrickson G H, Chmelka B F, Stucky G D. Triblock copolymer syntheses of mesoporous silica with periodic 50 to 300 A pores. Science, 1998, 279: 548-552

7 Ying J Y, Mehnert C P, Wong M S. Synthesis and applications of supramolecular-templated mesoporous materials. Angew Chem Int Ed, 1999, 38: 56-77

8 Wan Y, Shi Y F, Zhao D Y. Designed synthesis of mesoporous solids via nonionic-surfactant-templating approach. Chem Commun, 2007, 897-926

9 Schüth F. Non-siliceous mesostructured and mesoporous materials. Chem Mater, 2001, 13: 3184-3195

10 He X, Antonell D. Recent advances in synthesis and applications of transition metal containing mesoporous molecular sieves. Angew Chem Int Ed, 2002, 41: 214-229

11 Wan Y, Yang H F, Zhao D Y. "Host-guest" chemistry in the synthesis of ordered nonsiliceous mesoporous materials. Acc Chem Res, 2006, 39: 423-432

12 Boettcher S W, Fan J, Tsung C K, Shi Q, Stucky G D. Harnessing the sol-gel process for the assembly of non-silicate mesostructured oxide materials. Acc Chem Res, 2007, 40: 784-792

13 Yang P D, Zhao D Y, Margolese D I, Chmelka B F, Stucky G D. Generalized syntheses of large-pore mesoporous metal oxides with semicrystalline frameworks. Nature, 1998, 396: 152-155

14 Yuan Q, Liu Q, Song W G, Feng W, Pu W L, Sun L D, Zhang Y W, Yan C H. Ordered mesoporous Ce $\mathrm{e}_{1-x} \mathrm{Zr}_{x} \mathrm{O}_{2}$ solid solutions with crystalline walls. J Am Chem Soc, 2007, 129: 6698-6699

15 Yuan Q, Yin A X, Luo C, Sun L D, Zhang Y W, Duan W T, Liu H C, Yan C H. Facile synthesis for ordered mesoporous $\gamma$-aluminas with high thermal stability. J Am Chem Soc, 2008, 130: 3465-3472

16 Li L L, Duan W T, Yuan Q, Li Z X, Duan H H, Yan C H. Hierarchical $\gamma-\mathrm{Al}_{2} \mathrm{O}_{3}$ monoliths with highly ordered $2 \mathrm{D}$ hexagonal mesopores in macroporous walls. Chem Commun, 2009, 6174-6176

17 Attard G S, Glyde J C, Göltner C G. Liquid-crystalline phases as templates for the synthesis of mesoporous silica. Nature, 1995, 378: 366-368

18 Monnier A, Schüth F, Huo Q, Kumar D, Margolese D, Maxwell R S, Stucky G D, Krishnamurty M, Petroff P, Firouzi A, Janicke M, Chmelka B. Cooperative formation of inorganic-organic interfaces in the synthesis of silicate mesostructures. Science, 1993, 261: 1299-1303

19 Scott B J, Wirnsberger G, Stucky G D. Mesoporous and mesostructured materials for optical applications. Chem Mater, 2001, 13: $3140-3150$

20 Moller K, Bein T. Inclusion chemistry in periodic mesoporous hosts. Chem Mater, 1998, 10: 2950 - 2963

21 Kickelbick G. Hybrid inorganic-organic mesoporous materials. Angew Chem Int Ed, 2004, 43: 3102-3104

22 Hoffmann F, Cornelius M, Morell J, Fröba M. Silica-based mesoporous organic-inorganic hybrid materials. Angew Chem Int Ed, 2006, 45: 3216-3251

23 Sanchez C, Boissière C, Grosso D, Laberty C, Nicole L. Design, synthesis, and properties of inorganic and hybrid thin films having periodically organized nanoporosity. Chem Mater, 2008, 20: 682-737

24 Hunks W J, Ozin G A. Challenges and advances in the chemistry of periodic mesoporous organosilicas (PMOs). J Mater Chem, 2005, 15: $3716-3724$

25 Hatton B, Landskron K, Whitnall W, Perovic D, Ozin G A. Past, present, and future of periodic mesoporous organosilicass-The PMOs. Acc Chem Res, 2005, 38: 305-312

26 Fujita S, Inagaki S. Self-organization of organosilica solids with molecular-scale and mesoscale periodicities. Chem Mater, 2008, 20: 891-908

27 Fan H, Lu Y, Stump A, Reed S T, Baer T, Schunk R, Perez-Luna V, López G P, Brinker C J. Rapid prototyping of patterned functional nanostructures. Nature, 2000, 405: 56-60

28 Wirnsberger G, Scott B J, Stucky G D. pH sensing with mesoporous thin films. Chem Commun, 2001, 119-120 
29 Liu N G, Chen Z, Dunphy D R, Jiang Y B, Assink R A, Brinker C J. Photoresponsive nanocomposite formed by self-assemly of an azobenzene-modified silane. Angew Chem Int Ed, 2003, 42: 1731-1734

30 Li L L, Fang C J, Yuan Q, Yan C H. Stabilization of facial isomer of tris(8-hydroxyquinolinate) aluminum though confinement in silica-surfactant mesostructures. Appl Phys Lett, 2007, 90, 231908

31 Mal N K, Fujiwara M, Tanaka Y. Photocontrolled reversible release of guest molecules from coumarin-modified mesoporous silica. Nature, 2003, 421: 350-353

32 Nguyen T D, Leung K C F, Liong M, Liu Y, Stoddart J F, Zink J I. Versatile supramolecular nanovalves reconfigured for light activation. Adv Funct Mater, 2007, 17: 2101-2110

33 Angelos S, Johansson E, Stoddart J F, Zink J I. Mesostructured silica supports for functional materials and molecular machines. Adv Funct Mater, 2007, 17: 2261-2271

34 Zhang R, Ding W, Tu B, Zhao D. Mesoporous silica: An efficient nanoreactor for liquid-liquid biphase reactions. Chem Mater, 2007, 19: 4379-4381

35 Inagaki S, Guan S, Fukushima Y, Oshuna T, Terasaki O. Novel mesoporous materials with a uniform distribution of organic groups and inorganic oxide in their frameworks. J Am Chem Soc, 1999, 121: 9611-9614

36 Asefa T, MacLachlan M J, Coombs N, Ozin G A. Periodicmesoporous organosilicas with organic groups inside the channelwalls. Nature, 1999, 402: $867-871$

37 Melde B J, Holland B T, Blanford C F, Stein A. Mesoporous sieves with unified hybrid inorganic/organic frameworks. Chem Mater, 1999, 11: 3302-3308

38 Inagaki S, Guan S, Ohsuna T, Terasaki O. An orderedmesoporous organosilica hybrid material with crystal-like wall structure. Nature, 2002, 416: 304-307

39 Kapoor M P, Yang Q, Inagaki S. Self-assembly of biphenylene-bridged hybrid mesoporous solid with molecular-scale periodicity in the pore walls. J Am Chem Soc, 2002, 124: 15176-15177

40 Hossain K Z, Mercier L. Intra framework metal ion adsorption in ligand-functionalized mesoporous silica. Adv Mater, 2002, 14: 1053-1056

41 Olkhovyk O, Jaroniec M. Periodic mesoporous organosilica with large heterocyclic bridging groups, J Am Chem Soc, 2005, 127: 60-61

42 Peng H, Tang J, Yang L, Pang J, Ashbaugh H S, Brinker C J, Yang Z, Lu Y F. Responsive periodic mesoporous polydiacetylene/silica nanocomposites. J Am Chem Soc, 2006, 128: 5304-5305

43 Chandra D, Yokoi T, Tatsumi T, Bhaumik A. Highly luminescent organic-inorganic hybrid mesoporous silicas containing tunable chemosensor inside the pore wall. Chem Mater, 2007, 19: 5347-5354

44 Mizoshita N, Goto Y, Kapoor M P, Shimada T, Tani T, Inagaki S. Fluorescence emission from 2,6-naphthylene-bridged mesoporous organosilicas with an amorphous or crystal-like framework. Chem Eur J, 2009, 15: 219-226

45 Guo Y, Mylonakis A, Zhang Z, Yang G, Lelkes P I, Che S, Lu Q, Wei Y. Templated synthesis of electroactive periodic mesoporous organosilica bridged with oligoaniline. Chem Eur J, 2008, 14: 2909-2917

46 Whitnall W, Cademartiri L, Ozin G A. C60-PMO: Periodic mesoporous buckyballsilica. J Am Chem Soc, 2007, 129: 15644-15649

47 Polarz S, Kuschel A. Preparation of a periodically ordered mesoporous organosilica material using chiral building blocks. Adv Mater, 2006, 18: 1206-1209

48 García R A, Grieken R, Iglesias J, Morales V, Gordillo D. Synthesis of chiral periodic mesoporous silicas incorporating tartrate derivatives in the framework and their use in asymmetric sulfoxidation. Chem Mater, 2008, 20: 2964-2971

49 Dufaud V, Beauchesne F, Bonneviot L. Organometallic chemistry inside the pore walls of mesostructured silica materials. Angew Chem Int Ed, 2005, 44: 3475-3477

50 Hudson S, Cooney J, Hondnett B K, Magner E. Chloroperoxidase on periodic mesoporous organosilanes: immobilization and reuse. Chem Mater, 2007, 19: 2049-2055

51 Lu Y F, Yang Y, Sellinger A, Lu M C, Huang J M, Fan H Y, Haddad R, Lopez G, Burns A R, Sasaki D Y, Shelnutt J, Brinker C J. Self-assembly of mesoscopically ordered chromatic polydiacetylene/silica nanocomposites. Nature, 2001, 410: 913 - 917

52 Yang Y, Lu Y F, Lu M C, Huang J M, Haddad R, Xomeritakis G, Liu N G, Malanoski A P, Sturmayr D, Fan H Y, Sasaki D Y, Assink R A, Shelnutt J A, van Swol F, Lopez G, Burns A R, Brinker C J. Functional nanocomposites prepared by self-assembly and polymerization of diacetylene surfactants and silicic acid. J Am Chem Soc, 2003, 125: 1269-1277

53 Aida T, Tajima K. Photoluminescent silicate microsticks containing aligned nanodomains of conjugated polymers by sol-gel-based in situ polymerization. Angew Chem Int Ed, 2001, 4: 3803-3806

54 Ikegame M, Tajima K, Aida T. Template synthesis of polypyrrole nanofibers insulated within one-dimensional silicate channels: Hexagonal versus lamellar for recombination of polarons into bipolarons. Angew Chem Int Ed, 2003, 42: 2154-2157

55 Guo R, Li G, Zhang W, Shen G, Shen D. Superlong polypyrrole nanowires aligned within ordered mesoporous silica channels. 
ChemPhysChem, 2005, 6: 2025-2028

56 Li G, Bhosale S, Wang T, Zhang Y, Zhu H, Fuhrhop J H. Gram-scale synthesis of submicrometer-long polythiophene wires in mesoporous silica matrices. Angew Chem Int Ed, 2003, 42: 3818-3821

57 Yang Z, Kou X, Ni W, Sun Z, Li L, Wang J. Fluorescent mesostructured polythiophene-silica composite particles synthesized by in situ polymerization of structure-directing monomers. Chem Mater, 2007, 19: 6222-6229

58 Clark A P Z, Shen K F, Rubin Y F, Tolbert S H. An amphiphilic poly(phenylene ethynylene) as the structure-directing agent for periodic nanoscale silica composite materials. Nano Lett, 2005, 5: 1647-1652

59 Li L L, Fang C J, Sun H, Yan C H. Hierarchical self-assembly of pH-responsive nanocomposites with molecular-scale and mesoscale periodicities. Chem Mater, 2008, 20: 5977-5986

60 Li L L, Sun H, Bai Y C, Fang C J, Yan C H. Orientational organization of organic semiconductors within periodic nanoscale silica channels: Modification of fluorophore photophysics through hierarchical self-assembly. Chem Eur J, 2009, 15: 4716-4724

61 Li L L, Sun H, Yuan Q, Fang C J, Sun L D, Yan C H. Mesostructured hybrids containing potential donors and acceptors with molecular-scale and meso-scale segregation and ordering: toward the development of smart materials through hierarchical self-assembly. Chem Mater, 2009, 21: 4589-4597

62 Bhongale C J, Hsu C S. Emission enhancement by formation of aggregates in hybrid chromophoric surfactant amphiphile/silica nanocomposites. Angew Chem Int Ed, 2006, 45: 1404-1408

63 Yang C H, Bhongale C J, Liao Y M, Hsu C S. Fabrication of hybrid chromophoric amphiphile/silica nanocomposite-based light emitting devices with enhanced performance. J Mater Chem, 2007, 17: 243-253

64 Sofos M, Goldberger J, Stone D A, Allen J E, Ma Q, Herman D J, Tsai W W, Lauhon L J, Stupp S I. A synergistic assembly of nanoscale lamellar photoconductor hybrids. Nat Mater, 2009, 8: 68-75

\title{
Functional-template directed self-assembly (FTDSA) of mesostruc- tured organic-inorganic hybrid materials
}

\author{
LI LeLe, SUN LingDong, ZHANG YaWen \& YAN ChunHua*
}

Beijing National Laboratory for Molecular Sciences, State Key Lab of Rare Earth Materials Chemistry and Applications \& PKU-HKU Joint Lab in Rare Earth Materials and Bioinorganic Chemistry, College of Chemistry and Molecular Engineering, Peking University, Beijing 100871, China

\begin{abstract}
Since the discovery of a surfactant directed self-assembly approach for the fabrication of mesoporous silica in 1992, increasing attention has been focused on the design and synthesis of mesostructured functional materials. Organic functionalization is becoming a major topic in this research field, since highly ordered mesostructured organic-inorganic hybrids offer novel functionalities and enhanced performance over their individual components. We begin with a brief overview of the three fundamental methods (post-synthetic grafting technique, co-condensation method, and preparation of periodic mesoporous organosilicas) for the preparation of organically functionalized mesostructured silicas. The main focus will be on one of the most promising approaches, which herein was named as functional-template directed self-assembly (FTDSA) approach that in the eyes of the authors has a special position in preparation of this class of hybrid materials. A comprehensive overview of the state of research in the area of FTDSA and their potential applications will be given.
\end{abstract}

Keywords: mesoporous materials, self-assembly, organic-inorganic hybrids 